\title{
Nature of beginning
}

\author{
J.C. Hodge ${ }^{1 *}$ \\ ${ }^{1}$ Retired, 477 Mincey Rd., Franklin, NC, 28734
}

\begin{abstract}
The Big Bang's model of the beginning of our universe is inconsistent with all other forms of beginning in the universe. The Scalar Theory of Everything (STOE) posits the general principles of all the components of the universe also apply to the universe as a whole. Therefore, the universe began as a single source, grew, and multiplied.
\end{abstract}

keywords: STOE, Theory of Everything, QSSC

\section{Introduction}

The Scalar Theory of Everything (STOE) posits the general principles of all the components of the universe also apply to the universe as a whole. This principle was used to relate the fine-tuning of parameters in the universe and the CMB temperature of the universe (Hodge 2006).

The major difference between the standard Big Bang cosmology (BB) model and the Quasi-Steady State Cosmology (QSSC) model is that matter creation of a "C-field" occurs continuously in the center of galaxies (Sources) with energy conservation. The resulting ejection increases the volume of the universe such that the Perfect Cosmological Principle (the universe looks the same in density and time) applies. The QSSC is a Machian theory. The basic observations that are currently interpreted as supporting the BB can be interpreted as supporting the QSSC, also (Hoyle et al. 2000, p. 321). The QSSC suggests no initial singularity. The creation field controls the expansion of the universe such that the density remains approximately constant with periods of expansion and contractions. The physical control mechanism is unexplained. Although debated, the QSSC has a better model than BB for QSOs [data from Arp (1998)], for the large scale structure of the universe, and for the abundance of light elements.

Both the BB and QSSC have many unexplained cosmological anomalies and neither suggests correspondence with the world of Quantum Mechanics (the double slit experiment). The STOE is a model that starts with the QSSC, that adds Sink galaxies, and that suggests two components of the stuff of the universe emerge into the universe.

*E-mail: jchodge@frontier.com 


\section{REFERENCES}

Arp (1998) suggested Seyfert galaxies are Quasar factories and were much closer than redshift values indicated. That is, QSOs are daughter galaxies and are growing to become new galaxies.

This paper suggests the nature of beginning of life, of planets, of suns, and of galaxies is also the nature of the beginning of the universe.

The physics observations of life include a beginning and an end. The life of a bacterial cell begins with cell division. The half-cell then gathers material from the environment to become a full-cell which then reproduces to form more cells.

Multi-cellular beings begin by half-cells uniting to form a single cell of the new being. The new cell then gathers material from the environment to become a new being which then reproduces to form more, similar beings.

Hydrogen in the outer regions of a spiral galaxy combine to form stars which then move inward. Planets start from small chunks of matter that combine with other asteroids.

The QSSC and the STOE suggest the universe began as a single source of the components of the universe and grew by the matter forming around the source to form the spiral galaxy. The number of sources reproduced by a method suggested by Halton Arp (1998). The STOE suggest some of the matter escapes the spiral galaxy and condenses to form sinks of elliptical galaxies in galaxy clusters. Thus, the difference between spiral and elliptical galaxies is explained (Binney, J. \& Merrifield, M. 1998).

The STOE suggest this pattern of starting small and growing is repeated for the universe as a whole. Therefore, the concept of starting big and then running down of the Big Bang universe model is incorrect.

\section{References}

Arp, H., 1998, Seeing Red: redshifts, cosmology and academic science, (ISBN 0-9683689-0-5, Apeiron, Montreal, Canada)

Binney, J. \& Merrifield, M., 1998, Galactic Astronomy, (ISBN 0-691-00402-1, Princeton Univ. Press, Princeton, NJ, USA).

Hoyle, F., Burbidge, G., \& Narklikar, J.V., 2000, A different approach to cosmology, (ISBN 9781469987361, Cambridge Univ. Press, Cambridge).

Narklikar, J.V. et al. 2015. Mon. Not. Astron. Soc. Physics Letters A 250, 1.

Hodge, J.C., 2006, Scalar potential model of the CMB radiation temperature, arXiv:astro-ph/0603140.

Hodge, J.C., 2018, STOE replaces relativity and quantum mechanics, (ISBN 978-613-9-91465-4, available through Amazon.com). 